\title{
Risk of Febrile Neutropenia Associated With Select Myelosuppressive Chemotherapy Regimens in a Large Community-Based Oncology Practice
}

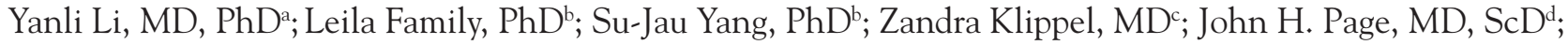
and Chun Chao, $\mathrm{PhD}^{\mathrm{b}}$

\begin{abstract}
Background: NCCN has classified commonly used chemotherapy regimens into high (>20\%), intermediate $(10 \%-20 \%)$, or low (<10\%) febrile neutropenia (FN) risk categories based primarily on clinical trial evidence. Many chemotherapy regimens, however, remain unclassified by NCCN or lack FN incidence data in real-world clinical practice. Patients and Methods: We evaluated incidence proportions of FN and grade 4 and 3/4 neutropenia during the first chemotherapy course among patients from Kaiser Permanente Southern California who received selected chemotherapy regimens without well-established FN risk. Patients given granulocyte colony-stimulating factor (G-CSF) prophylaxis were excluded. Sensitivity analyses were performed to account for FN misclassification and censoring. Results: From 2008 to 2013 , $1,312$ patients with breast cancer who received docetaxel and cyclophosphamide (TC; $n=853)$ or docetaxel, carboplatin, and trastuzumab $(\mathrm{TCH} ; \mathrm{n}=459) ; 1,321$ patients with colorectal cancer who received capecitabine and oxaliplatin (XELOX; n=401) or leucovorin, 5-fluorouracil, and oxaliplatin (FOLFOX6; n=920); 307 patients with non-Hodgkin's lymphoma who received bendamustine with or without rituximab; and 181 patients with multiple myeloma who received lenalidomide with or without dexamethasone were included. Crude FN risk was $>20 \%$ for both breast cancer regimens (TC and TCH). Crude FN risks for XELOX, FOLFOX6, bendamustine, and lenalidomide were $<10 \%$; however, when potential FN misclassification and censoring were considered, FN risks were $>10 \%$. Conclusions: Our results support published literature highlighting the real-world, "high" FN risk of the TC and TCH regimens for breast cancer. There is strong suggestive evidence that FN risks for XELOX, FOLFOX6, bendamustine, and lenalidomide are $>10 \%$. Calculation of chemotherapy course-level FN incidence without controlling for differential censoring for patients who discontinued regimens early, or possible FN misclassification, might have resulted in bias toward an underestimation of the true FN risk. These findings help define FN risk of the selected regimens in the real-world setting and inform prophylactic G-CSF use.
\end{abstract}

J Natl Compr Canc Netw 2017;15(9):1122-1130 doi: 10.6004/jncen.2017.7010

\section{Background}

Febrile neutropenia (FN) is a serious adverse effect of myelosuppressive chemotherapy that can affect treatment by contributing to dose delays and reductions. ${ }^{1} \mathrm{FN}$ often requires hospitalization and incurs a significant healthcare cost. ${ }^{2}$ Prophylactic use of recombinant human granulocyte colony-stimulating factors (G-CSFs),

From ${ }^{a}$ Center for Observational Research, Amgen Inc., South San Francisco; ${ }^{b}$ Department of Research and Evaluation, Kaiser Permanente Southern California, Pasadena; and 'Hematology/Oncology, and dCenter for Observational Research, Amgen Inc., Thousand Oaks, California. Submitted October 26, 2016; accepted for publication May 8, 2017. Drs. Li, Klippel, and Page have disclosed that they are employees of and stockholders in Amgen Inc. Drs. Family, Yang, and Chao have disclosed that they have received research funding from Amgen Inc. such as filgrastim or pegfilgrastim, can significantly reduce FN risk and FN-related costs. ${ }^{3-5}$

The risk of developing FN depends on patient and disease characteristics and the myelotoxicity of chemotherapy regimens. ${ }^{6}$ Understanding the FN risk associated with individual chemotherapy regimens can help guide prophylactic G-CSF use. The NCCN Clinical Practice

This study was funded by Amgen Inc.

Author contributions: Study conception and supervision: Li, Chao, Page. Development of study design: all authors. Conduct of analyses: Yang. Interpretation of results: all authors. Preparation of manuscript: Li, Family. Review of, substantive input on, and approval of manuscript: all authors. Correspondence: Chun Chao, PhD, Kaiser Permanente Southern California, Department of Research and Evaluation, 100 South Los Robles Avenue, 2nd Floor, Pasadena, CA 91101. E-mail: chun.r.chao@kp.org 
FN Risk for Select Chemotherapy Regimens

Guidelines in Oncology (NCCN Guidelines) for Myeloid Growth Factors ${ }^{7}$ classified some commonly used chemotherapy regimens into FN-risk groups (high [>20\%], intermediate [10\%-20\%], or low $[<10 \%]$ ) based primarily on $\mathrm{FN}$ incidence throughout the chemotherapy course reported in clinical trials. Primary prophylactic G-CSF (ppG-CSF) use is recommended in patients receiving high-risk chemotherapy regimens and should be considered for those who receive intermediate-risk regimens and have additional risk factors. ${ }^{\text {? }}$

The chemotherapy landscape for cancer treatment continues to evolve, and many new therapeutic agents and combinations have recently become available. FN risks for these new regimens are either not classified by NCCN Guidelines or their FN-risk profiles in real-world clinical practice are not fully understood. Assessing FN risk for chemotherapy regimens outside of clinical trial settings is of great importance; previous studies have noted differences in FN risks reported in clinical trials versus actual clinical practice. ${ }^{8-10}$ The differences may be explained by the more stringent enrollment criteria of clinical trials and different standards of care in clinical trials. Additionally, G-CSF use (including ppG-CSF) is sometimes allowed in clinical trials.

Using data from Kaiser Permanente Southern California (KPSC), we evaluated FN risks associated with docetaxel/cyclophosphamide (TC) and docetaxel/carboplatin/trastuzumab (TCH) for breast cancer; leucovorin/5-fluorouracil/oxaliplatin (FOLFOX6) or capecitabine/oxaliplatin (XELOX) for colorectal cancer (CRC); bendamustine with or without rituximab $(\mathrm{B} \pm \mathrm{R})$ for non-Hodgkin's lymphoma (NHL); and lenalidomide with or without dexamethasone for multiple myeloma (MM). When this study was initiated, TC was established by the NCCN Myeloid Growth Factors Guidelines ${ }^{7}$ as a regimen with intermediate $\mathrm{FN}_{\text {risk }}{ }^{1}$; however, substantial variability in FN risk has been seen outside of clinical trials. ${ }^{11,12}$ Additionally, TCH had not been classified by NCCN. Both regimens are now classified as high-risk FN regimens in the current guidelines. ${ }^{13-15}$ FOLFOX has been classified as an intermediate-risk regimen based on clinical trial findings for FOLFOX4. ${ }^{7,16} \mathrm{FN}$ risk for FOLFOX6, an alternative regimen with a more simplified administration of 5-fluorouracil infusion, ${ }^{17}$ remains unclassified. All other regimens evaluated have not been classified by NCCN for FN risk and have limited real-world data.

\section{Patients and Methods}

\section{Study Population}

KPSC is an integrated managed care organization that provides comprehensive health services for 3.5 million enrollees with diverse racial, ethnic, and socioeconomic backgrounds who broadly represent the residents of southern California. ${ }^{18}$ KPSC maintains electronic health records for most aspects of care, including diagnoses, medical procedures, pharmacy dispensing, laboratory test results, and disease registries. Incident cancer cases were identified using KPSC's SEER-affiliated cancer registry.

We identified a retrospective cohort of adult patients diagnosed with breast cancer, CRC, NHL, or MM at KPSC between January 1, 2008, and December 31, 2013, who initiated a new course of chemotherapy with one of the selected chemotherapy regimens before June 30, 2014. Patients with $<12$ months of KPSC membership, unknown cancer stage, or multiple primary cancers before chemotherapy initiation, or who had received bone marrow transplant, stem cell transplant, or radiation therapy before or during the chemotherapy course under study were excluded, as were those who received prophylactic G-CSF within 5 days of chemotherapy initiation in the first cycle. Additionally, patients were censored at subsequent cycles in which prophylactic G-CSF was used within 5 days of chemotherapy initiation.

\section{Exposure Assessment}

The first chemotherapy course with one of the selected chemotherapy regimens under study among eligible patients was included in the analysis. A chemotherapy course was defined as sequential administrations of chemotherapy agents separated by a gap of $<60$ days. The start of the chemotherapy course was defined as the date of initial chemotherapy administration, and the end date was defined as the last date of administration of selected chemotherapy regimens with a subsequent gap of $\geq 60$ days. Information on the chemotherapy agent, dose, cycle length, and number of cycles administered was obtained from KPSC's chemotherapy infusion databases (Table 1). 
Li et al

\begin{tabular}{|c|c|c|c|c|c|}
\hline $\begin{array}{l}\text { Cancer } \\
\text { Type }\end{array}$ & Regimen & Acronym & Standard Dose ${ }^{a}$ & $\begin{array}{c}\text { Maximum Cycles } \\
\text { Studied, } \mathbf{n}^{\mathrm{b}}\end{array}$ & $\begin{array}{c}\text { Cycle } \\
\text { Length, days }\end{array}$ \\
\hline Breast & Docetaxel and cyclophosphamide & TC & $\begin{array}{l}\text { Docetaxel, } 75 \mathrm{mg} / \mathrm{m}^{2}+\text { cyclophosphamide, } 600 \\
\mathrm{mg} / \mathrm{m}^{2} \text { on day } 1\end{array}$ & 6 & 21 \\
\hline Breast & $\begin{array}{l}\text { Docetaxel, carboplatin, and } \\
\text { trastuzumab }\end{array}$ & $\mathrm{TCH}$ & Docetaxel, $75 \mathrm{mg} / \mathrm{m}^{2}+$ carboplatin, AUC6 on day 1 & 6 & 21 \\
\hline CRC & Capecitabine and oxaliplatin & XELOX & $\begin{array}{l}\text { Capecitabine, } 1,700-2,000 \mathrm{mg} / \mathrm{m}^{2} \text { daily, day } 1-14+ \\
\text { oxaliplatin, } 130 \mathrm{mg} / \mathrm{m}^{2} \text { on day } 1\end{array}$ & 8 & 21 \\
\hline CRC & $\begin{array}{l}\text { Leucovorin, fluorouracil, and } \\
\text { oxaliplatin }\end{array}$ & FOLFOX6 & $\begin{array}{l}\text { Leucovorin, } 400 \mathrm{mg} / \mathrm{m}^{2} \text { IV over } 2 \mathrm{~h} \text { before } 5-\mathrm{FU} \text { on } \\
\text { day } 1+5-\mathrm{FU}, 400-\mathrm{mg} / \mathrm{m}^{2} \text { bolus then } 1,200 \mathrm{mg} / \mathrm{m}^{2} \\
\text { for } 2 \text { days }+ \text { oxaliplatin, } 85 \mathrm{mg} / \mathrm{m}^{2} \text { on day } 1\end{array}$ & 12 & 14 \\
\hline NHL & Bendamustine \pm rituximab & $B \pm R$ & Bendamustine, $90 \mathrm{mg} / \mathrm{m}^{2}$ on days 1 and 2 & 6 & 28 \\
\hline MM & Lenalidomide \pm dexamethasone & N/A & Lenalidomide, $25 \mathrm{mg}^{\mathrm{d}}$ on days $1-21$ & $6-9$ & 28 \\
\hline
\end{tabular}

Abbreviations: 5-FU, 5-fluorouracil; AUC, area under the time-concentration curve; CRC, colorectal cancer; IV, intravenous; MM, multiple myeloma; N/A, not applicable; NHL, non-Hodgkin's lymphoma.

anly standard doses for myelosuppressive chemotherapy agents are shown in the table.

${ }^{\mathrm{b}}$ Maximal numbers of cycles included in the evaluation are based on standard numbers of cycles for each regimen reported in literature.

cPatients who received weekly regimens of FOLFOX6 or did not receive 5-FU bolus were excluded.

dOnly patients receiving lenalidomide, $25 \mathrm{mg}$ were included in the current analysis in an attempt to capture initial therapy.

\section{Outcome Assessment}

The primary end point, FN, was defined using one of the following methods: (1) neutropenia (ICD-9 code 288.0) and fever (ICD-9 code 780.6) within 7 days of hospitalization; (2) absolute neutrophil count $(\mathrm{ANC})<1,000 / \mathrm{mcL}$ and fever (ICD-9 code 780.6) within 7 days of hospitalization; (3) hospitalization with neutropenia (ICD-9 code 288.0) as the primary diagnosis; or (4) neutropenia (ICD-9 code 288.0) or ANC $<1,000 / \mathrm{mcL}$ within 7 days of hospitalization with an ICD-9 code for bacterial/fungal infection. Neutropenia during a chemotherapy cycle usually lasts 1 to 5 days $^{19,20}$; consequently, there is a high probability that such transient changes in neutrophil counts might not be detected when testing is performed, even when a patient has an infection. Furthermore, intravenous antibiotics are most often given when there is at least a strong suspicion of infection. To account for possible FN misclassification, we conducted a sensitivity analysis; FN cases were defined as occurring in patients who either met the aforementioned FN definition or received treatment with intravenous antibiotics during the chemotherapy course. The secondary end points were grade 4 (ANC $<500 / \mathrm{mcL}$ ) or $3 / 4$ neutropenia (ANC $<1,000 / \mathrm{mcL}$ ).

Follow-up of each patient to ascertain end points of interest began at the first chemotherapy administration with a selected regimen and ended either when the chemotherapy regimen was switched, at 28 days after initiation of the last cycle, at termination of KPSC membership, or at death, whichever came first. Furthermore, if gaps between 2 adjacent cycles were $>28$ days, the end points of interest for the earlier cycle were captured only within 28 days after initiating the cycle.

\section{Covariates of Interest}

Information on patients' age, sex, race/ethnicity, and cancer stage at diagnosis was obtained from KPSC's cancer registry. Information was also collected on selected comorbidities previously shown to be related to $\mathrm{FN}$ risk, ${ }^{21,22}$ including diabetes, hypertension, cardiovascular diseases, chronic obstructive pulmonary disorder/emphysema, liver disease, renal disease, autoimmune conditions, thyroid disorder, peptic ulcer disease, connective disease, and HIV infection. All available records in KPSC's disease registry were used to evaluate the history of cancer, HIV, and diabetes. The presence of other conditions was assessed using ICD-9 diagnosis codes within the 12 months before chemotherapy initiation.

\section{Statistical Analysis}

Descriptive analyses were conducted to characterize demographics, disease characteristics, comorbidities, and doses used in the first chemotherapy cycle per body surface area for each of the selected chemotherapy regimens. Course- and cycle-level crude incidence proportions of study end points were calculated (defined as the number of patients who developed the study end points throughout the course of chemotherapy or in a given cycle divided by the number of eligible patients in the 
FN Risk for Select Chemotherapy Regimens

beginning of the chemotherapy course or cycle). The 95\% CIs for the crude incidence proportions were estimated using normal approximation. To control for possible differences in characteristics of patients with cancer receiving select regimens relative to all chemotherapy-treated patients with the same cancer type, standardized incidence proportions were estimated using a model-based standardization approach adjusting for age, sex, race/ethnicity, and cancer stage. All KPSC members diagnosed with a cancer of interest who received any chemotherapy during the study period were used as the reference group to calculate standardized incidence proportions. The 95\% CIs for all standardized incidence proportions were calculated using 1,000 bootstrap samples and the percentile method. In addition, chemotherapy course-level FN incidence proportions by age group $(<65$ or $\geq 65$ years) and by number of comorbidities $(0,1, \geq 2)$ were calculated.

Because our data were significantly affected by censoring, we performed a sensitivity analysis to explore the upper bounds for the true FN incidence proportions under different censoring scenarios. This was accomplished by estimating the FN incidence proportions by the number of chemotherapy cycles completed. FN incidence proportion was estimated separately for patients who completed 1 cycle only, for patients who completed 2 cycles only, and so forth, and for patients who completed the maximal number of cycles evaluated.

A substantial proportion of patients with breast cancer were excluded from the final study cohort because of the use of ppG-CSF; consequently, we performed a sensitivity analysis among patients with breast cancer who met all other selection criteria but received ppG-CSF. This allowed for the capture of differences in $\mathrm{FN}$ incidence proportions between those who received ppG-CSF versus those who did not, and the comparison of these estimates to the literature.

\section{Results}

\section{Patients}

After applying the study inclusion/exclusion criteria, 1,312 patients with breast cancer (TC, $n=853$; $\mathrm{TCH}, \mathrm{n}=459)$, 1,321 with CRC (XELOX, n=401; FOLFOX6, $n=920$ ), 307 with NHL receiving $\mathrm{B} \pm \mathrm{R}$, and 181 with $\mathrm{MM}$ receiving lenalidomide with or without dexamethasone were included in the analysis. The population dispositions after applying each inclusion/exclusion criterion for the selected chemotherapy regimens are shown in supplemental eFigures 1-4 (available with this article at JNCCN.org). Of note, a high proportion of patients with breast cancer who met other selection criteria were excluded because of ppG-CSF use (TC, 27\% [n=320/1,177]; TCH, 23\% [n=134/593]).

Demographics and clinical characteristics of the study population by chemotherapy regimen are shown in Table 2. The mean age at diagnosis ranged from 55.5 to 66.9 years. The breast cancer regimens were mostly used in patients with early-stage disease (stage I or II), whereas most CRC and NHL regimens were given to patients with advanced-stage disease (stage III or IV). Table 2 also shows the distribution of doses at cycle 1 by chemotherapy agent. Demographics and clinical characteristics of patients with breast cancer who met other selection criteria but were excluded from the main analysis due to ppGCSF are shown in supplemental eTable 1.

\section{End Points}

The crude and standardized incidence proportions for FN, FN plus intravenous antibiotic use, and grade 4 and 3/4 neutropenia in the first chemotherapy course are presented in Table 3 . Incidence proportions of $\mathrm{FN}$ by each chemotherapy cycle and during the chemotherapy course by number of completed cycles are presented in supplemental eTables 2 and 3.

During the first 6 cycles of chemotherapy, 20.9\% and $25.1 \%$ of patients with breast cancer who received TC and TCH regimens, respectively, experienced $\geq 1$ FN episode (Table 3; supplemental eTable 2). When intravenous antibiotic treatment was included as part of the FN definition, $23.3 \%$ and $31.6 \%$ of patients with breast cancer who received the TC and $\mathrm{TCH}$ regimens, respectively, experienced FN (Table 3). Standardized incidence proportions were similar to the crude estimates. In the sensitivity analysis, we observed that patients who completed fewer cycles of the TC or TCH regimen had higher FN incidence proportions. The results demonstrate possible upper bounds for the FN incidences under different censoring scenarios (supplemental eTable 3). Additionally, $8.1 \%$ of 320 otherwise eligible patients with breast cancer who received TC and ppG-CSF 
Li et al

\begin{tabular}{|c|c|c|c|c|c|c|}
\hline & \multicolumn{2}{|c|}{ Breast } & \multicolumn{2}{|c|}{ CRC } & \multirow{2}{*}{$\frac{\mathrm{NHL}}{\substack{\mathrm{B} \pm \mathrm{R} \\
(\mathrm{n}=307)}}$} & \multirow{2}{*}{$\begin{array}{c}\text { MM } \\
\begin{array}{c}\text { Lenalidomide } \pm \\
\text { Dexamethasone } \\
(n=181)\end{array}\end{array}$} \\
\hline & $\begin{array}{c}\mathrm{TC} \\
(\mathrm{n}=853)\end{array}$ & $\begin{array}{c}\mathrm{TCH} \\
(\mathrm{n}=459)\end{array}$ & $\begin{array}{l}\text { XELOX } \\
(n=401)\end{array}$ & $\begin{array}{c}\text { FOLFOX6 } \\
(n=920)\end{array}$ & & \\
\hline Age at diagnosis, mean (SD) & $56.9(10.0)$ & $55.5(10.5)$ & $61.5(12.0)$ & $58.9(11.5)$ & $66.9(11.9)$ & $62.8(10.1)$ \\
\hline $18-39$ y, n (\%) & $29(3.4)$ & $32(7.0)$ & $19(4.7)$ & $47(5.1)$ & $4(1.3)$ & $1(0.6)$ \\
\hline $40-49$ y, n (\%) & $181(21.2)$ & $100(21.8)$ & $46(11.5)$ & $145(15.8)$ & $26(8.5)$ & $21(11.6)$ \\
\hline $50-59$ y, n (\%) & $293(34.4)$ & $158(34.4)$ & $98(24.4)$ & $264(28.7)$ & $43(14.0)$ & $45(24.9)$ \\
\hline $60-69$ y, n (\%) & $262(30.7)$ & $128(27.9)$ & $132(32.9)$ & $285(31.0)$ & $100(32.6)$ & $65(35.9)$ \\
\hline $70-79$ y, n (\%) & $80(9.4)$ & $40(8.7)$ & $81(20.2)$ & $159(17.3)$ & 91 (29.6) & $44(24.3)$ \\
\hline$\geq 80 y, n(\%)$ & $8(0.9)$ & $1(0.2)$ & $25(6.2)$ & $20(2.2)$ & $43(14.0)$ & $5(2.8)$ \\
\hline Female, n (\%) & $853(100)$ & $459(100)$ & $191(47.6)$ & $453(49.2)$ & $131(42.7)$ & $74(40.9)$ \\
\hline \multicolumn{7}{|l|}{ Ethnicity, n (\%) } \\
\hline White, non-Hispanic & $469(55.0)$ & $219(47.7)$ & $183(45.6)$ & $457(49.7)$ & $186(60.6)$ & $80(44.2)$ \\
\hline African American & $152(17.8)$ & $68(14.8)$ & 77 (19.2) & $139(15.1)$ & $26(8.5)$ & $35(19.3)$ \\
\hline Asian & $108(12.7)$ & $90(19.6)$ & $41(10.2)$ & $104(11.3)$ & $17(5.5)$ & $12(6.6)$ \\
\hline Hispanic & $123(14.4)$ & $76(16.6)$ & $91(22.7)$ & $211(22.9)$ & $73(23.8)$ & $46(25.4)$ \\
\hline Other & $1(0.1)$ & $6(1.3)$ & $9(2.2)$ & $9(1.0)$ & $5(1.6)$ & $8(4.4)$ \\
\hline \multicolumn{7}{|l|}{ Stage at diagnosis, n (\%) } \\
\hline 1 & $331(38.8)$ & $145(31.6)$ & $9(2.2)$ & $29(3.2)$ & $32(10.4)$ & NA \\
\hline II & $439(51.5)$ & $213(46.4)$ & $64(16.0)$ & $113(12.3)$ & $45(14.7)$ & NA \\
\hline III & $74(8.7)$ & $77(16.8)$ & $177(44.1)$ & $516(56.1)$ & $85(27.7)$ & NA \\
\hline IV & $9(1.1)$ & $24(5.2)$ & $151(37.7)$ & $262(28.5)$ & $145(47.2)$ & NA \\
\hline \multicolumn{7}{|c|}{ History of comorbidities of interest, $\mathrm{n}(\%)$} \\
\hline Diabetes & $112(13.1)$ & $81(17.7)$ & $80(20.0)$ & $182(19.8)$ & $66(21.5)$ & $38(21.0)$ \\
\hline Hypertension & $331(38.8)$ & $171(37.3)$ & $197(49.1)$ & $408(44.4)$ & $166(54.1)$ & $99(54.7)$ \\
\hline Cardiovascular disease $^{a}$ & $36(4.2)$ & $14(3.1)$ & $43(10.7)$ & $75(8.2)$ & $45(14.7)$ & $21(11.6)$ \\
\hline COPD/emphysema & $115(13.5)$ & $44(9.6)$ & $58(14.5)$ & $94(10.2)$ & $53(17.3)$ & $35(19.3)$ \\
\hline Moderate/severe liver disease ${ }^{\mathrm{b}}$ & $11(1.3)$ & $8(1.7)$ & $12(3.0)$ & $23(2.5)$ & $10(3.3)$ & $7(3.9)$ \\
\hline Moderate/severe renal disease & $59(6.9)$ & $28(6.1)$ & $33(8.2)$ & $69(7.5)$ & $51(16.6)$ & $15(8.3)$ \\
\hline 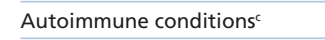 & $17(2.0)$ & $11(2.4)$ & $6(1.5)$ & $17(1.9)$ & $6(2.0)$ & $5(2.8)$ \\
\hline Thyroid disorder $^{d}$ & $119(14.0)$ & $47(10.2)$ & $37(9.2)$ & $82(8.9)$ & $41(13.4)$ & $13(7.2)$ \\
\hline Peptic ulcer disease & $3(0.4)$ & $0(0)$ & $10(2.5)$ & $12(1.3)$ & $6(2.0)$ & $3(1.7)$ \\
\hline Connective tissue disease & $17(2.0)$ & $13(2.8)$ & $3(0.8)$ & $11(1.2)$ & $7(2.3)$ & $4(2.2)$ \\
\hline HIV infection & $0(0)$ & $1(0.2)$ & $0(0)$ & $3(0.3)$ & $1(0.3)$ & $0(0)$ \\
\hline \multicolumn{7}{|l|}{ Number of comorbidities, $n(\%)$} \\
\hline 0 & $390(45.7)$ & $226(49.2)$ & $147(36.7)$ & $379(41.2)$ & $84(27.4)$ & $53(29.3)$ \\
\hline 1 & $226(26.5)$ & $116(25.3)$ & $112(27.9)$ & $260(28.3)$ & $91(29.6)$ & $57(31.5)$ \\
\hline 2 & $157(18.4)$ & $71(15.5)$ & $81(20.2)$ & $176(19.1)$ & $66(21.5)$ & $43(23.7)$ \\
\hline$\geq 3$ & $80(9.4)$ & $46(10.0)$ & $61(15.2)$ & 105 (11.4) & $66(21.5)$ & $28(15.5)$ \\
\hline $\begin{array}{l}\text { Grade } 3 / 4 \text { neutropenia } \\
\text { within } 6 \text { months before first } \\
\text { chemotherapy, } n(\%)\end{array}$ & $14(1.6)$ & $15(3.3)$ & $49(12.2)$ & $87(9.5)$ & $48(15.6)$ & $25(13.8)$ \\
\hline \multicolumn{7}{|l|}{ Initial dose at cycle $1, \mathrm{mg} / \mathrm{m}^{2}$} \\
\hline Chemotherapy agent 1 & Docetaxel & Docetaxel & Oxaliplatin & Oxaliplatin & Bendamustine & Lenalidomide $^{e}$ \\
\hline Mean (SD) & $74.0(4.4)$ & $73.7(4.8)$ & $122.6(16.6)$ & $84.7(5.9)$ & $85.9(12.8)$ & - \\
\hline Range & $32.7-103.1$ & $35.8-81.6$ & $64.7-179.3$ & $41.5-128.5$ & $40.0-126.4$ & - \\
\hline Median & 75.0 & 75.0 & 130.0 & 85.0 & 90.0 & - \\
\hline $\mathrm{IQR}$ & $74.5-75.0$ & $74.7-75.0$ & $129.1-130$ & $84.9-85.0$ & $87.5-90.0$ & - \\
\hline Chemotherapy agent 2 & Cyclophosphamide & Carboplatin & Capecitabine & 5-FU & - & - \\
\hline Mean (SD) & $594.0(30.6)$ & $347.9(71.5)$ & $1,583.1(321.0)$ & $2,701.1(308.3)$ & - & - \\
\hline Range & $286.5-825.0$ & $85.3-575.8$ & $473.8-4,067.8$ & $970.9-3,500$ & - & - \\
\hline Median & 600.0 & 347.0 & $1,612.9$ & $2,800.0$ & - & - \\
\hline IQR & $598.9-600.0$ & $305.3-393.0$ & $1,445.1-1,741.3$ & $2,790.4-2,800.0$ & - & - \\
\hline
\end{tabular}

Abbreviations: 5-FU, 5-fluorouracil; $\mathrm{B} \pm \mathrm{R}$, bendamustine with or without rituximab; COPD, chronic obstructive pulmonary disease; CRC, colorectal cancer; FOLFOX6, leucovorin/5-FU/oxaliplatin; IQR, interquartile range; MM, multiple myeloma; NA, not available; NHL, non-Hodgkin's lymphoma; TC, docetaxel/cyclophosphamide; TCH, docetaxel/carboplatin/trastuzumab; XELOX, capecitabine/oxaliplatin. ancluded myocardial infarction, stroke, congestive heart failure, and peripheral vascular disease.

bIncluded hepatitis and cirrhosis.

Included rheumatoid arthritis, systemic lupus erythematosus, multiple sclerosis, and inflammatory bowel disease.

Included autoimmune thyroiditis.

eThe dose of lenalidomide in the first cycle was $25 \mathrm{mg}$ for every patient with MM. 
FN Risk for Select Chemotherapy Regimens

\begin{tabular}{|c|c|c|c|c|c|c|}
\hline & \multicolumn{2}{|c|}{ Breast } & \multicolumn{2}{|c|}{ CRC } & \multirow{2}{*}{$\frac{\mathrm{NHL}}{\substack{\mathrm{B} \pm \mathrm{R} \\
(\mathrm{n}=307)}}$} & \multirow{2}{*}{$\begin{array}{c}\text { MM } \\
\begin{array}{c}\text { Lenalidomide } \pm \\
\text { Dexamethasone } \\
(n=181)\end{array}\end{array}$} \\
\hline & $\begin{array}{c}\text { TC } \\
(n=853)\end{array}$ & $\begin{array}{c}\text { TCH } \\
(\mathrm{n}=459)\end{array}$ & $\begin{array}{l}\text { XELOX } \\
(n=401)\end{array}$ & $\begin{array}{c}\text { FOLFOX6 } \\
(\mathrm{n}=920)\end{array}$ & & \\
\hline \multicolumn{7}{|c|}{ Crude incidence proportion, $\%(95 \% \mathrm{Cl})$} \\
\hline $\mathrm{FN}$ & $\begin{array}{l}20.9 \\
(18.1-23.6)\end{array}$ & $\begin{array}{l}25.1 \\
(21.1-29.0)\end{array}$ & $\begin{array}{l}5.0 \\
(2.9-7.1)\end{array}$ & $\begin{array}{l}6.3 \\
(4.7-7.9)\end{array}$ & $\begin{array}{l}8.8 \\
(5.6-12.0)\end{array}$ & $\begin{array}{l}7.2 \\
(3.4-10.9)\end{array}$ \\
\hline $\mathrm{FN}+\mathrm{IV}$ antibiotics & $\begin{array}{l}23.3 \\
(20.5-26.2)\end{array}$ & $\begin{array}{l}31.6 \\
(27.3-35.8)\end{array}$ & $\begin{array}{l}14.0 \\
(10.6-17.4)\end{array}$ & $\begin{array}{l}14.2 \\
(12.0-16.5)\end{array}$ & $\begin{array}{l}18.9 \\
(14.5-23.3)\end{array}$ & $\begin{array}{l}16.0 \\
(10.7-21.4)\end{array}$ \\
\hline Grade 4 neutropenia & $\begin{array}{l}44.3 \\
(41.0-47.7)\end{array}$ & $\begin{array}{l}52.1 \\
(47.5-56.6)\end{array}$ & $\begin{array}{l}22.2 \\
(18.1-26.3)\end{array}$ & $\begin{array}{l}32.3 \\
(29.3-35.3)\end{array}$ & $\begin{array}{l}24.7 \\
(22.4-27.0)\end{array}$ & $\begin{array}{l}26.5 \\
(20.1-33.0)\end{array}$ \\
\hline Grade $3 / 4$ neutropenia & $\begin{array}{l}53.6 \\
(50.2-56.9\end{array}$ & $\begin{array}{l}62.8 \\
(58.3-67.2)\end{array}$ & $\begin{array}{l}27.2 \\
(22.8-31.5)\end{array}$ & $\begin{array}{l}47.0 \\
(43.7-50.2)\end{array}$ & $\begin{array}{l}29.6 \\
(24.5-34.8)\end{array}$ & $\begin{array}{l}35.9 \\
(28.9-43.4)\end{array}$ \\
\hline \multicolumn{7}{|c|}{ Standardized incidence proportion, \% (95\% Cl) } \\
\hline $\mathrm{FN}$ & $\begin{array}{l}20.2 \\
(17.3-23.2)\end{array}$ & $\begin{array}{l}25.2 \\
(21.0-29.1)\end{array}$ & $\begin{array}{l}5.1 \\
(3.0-7.5)\end{array}$ & $\begin{array}{l}6.7 \\
(5.2-8.3)\end{array}$ & $\begin{array}{l}7.5 \\
(4.8-10.5)\end{array}$ & $\begin{array}{l}6.5 \\
(3.2-10.0)\end{array}$ \\
\hline $\mathrm{FN}+\mathrm{IV}$ antibiotics & $\begin{array}{l}22.9 \\
(19.9-25.9)\end{array}$ & $\begin{array}{l}31.7 \\
(27.4-35.9)\end{array}$ & $\begin{array}{l}13.9 \\
(10.5-17.5)\end{array}$ & $\begin{array}{l}14.1 \\
(11.9-16.3)\end{array}$ & $\begin{array}{l}16.8 \\
(13.1-21.2)\end{array}$ & $\begin{array}{l}17.4 \\
(11.8-23.2)\end{array}$ \\
\hline Grade 4 neutropenia & $\begin{array}{l}44.6 \\
(41.1-48.3)\end{array}$ & $\begin{array}{l}52.1 \\
(47.6-56.8)\end{array}$ & $\begin{array}{l}23.2 \\
(18.7-27.6)\end{array}$ & $\begin{array}{l}32.5 \\
(29.6-35.6)\end{array}$ & $\begin{array}{l}23.1 \\
(18.4-28.3)\end{array}$ & $\begin{array}{l}26.4 \\
(20.3-32.4)\end{array}$ \\
\hline Grade $3 / 4$ neutropenia & $\begin{array}{l}54.5 \\
(51.0-58.1)\end{array}$ & $\begin{array}{l}62.7 \\
(58.2-67.0)\end{array}$ & $\begin{array}{l}28.1 \\
(23.2-32.8)\end{array}$ & $\begin{array}{l}46.5 \\
(43.3-49.7)\end{array}$ & $\begin{array}{l}27.7 \\
(22.8-32.9)\end{array}$ & $\begin{array}{l}35.6 \\
(28.1-42.5)\end{array}$ \\
\hline
\end{tabular}

Abbreviations: $\mathrm{B} \pm \mathrm{R}$, bendamustine with or without rituximab; CRC, colorectal cancer; FN, febrile neutropenia; FOLFOX6, leucovorin/5-fluorouracil/ oxaliplatin; IV, intravenous; MM, multiple myeloma; NHL, non-Hodgkin's lymphoma; TC, docetaxel/cyclophosphamide; TCH, docetaxel/carboplatin/ trastuzumab; XELOX, capecitabine/oxaliplatin.

and $6.0 \%$ of 134 of those who received $\mathrm{TCH}$ and ppG-CSF developed FN (data not shown).

FN incidence proportions were observed to be $<10 \%$ for patients with CRC treated with XELOX (5\%) and FOLFOX6 (6\%), NHL treated with $\mathrm{B} \pm \mathrm{R}$ $(9 \%)$, and MM treated with lenalidomide with or without dexamethasone (7\%; Table 3 and supplemental eTable 2); however, the incidence proportions of patients who met the FN definition or received intravenous antibiotic treatment were $>10 \%$ for all of these regimens (Table 3). Results from additional sensitivity analysis also suggested that patients who completed a smaller number of cycles of $\mathrm{B} \pm \mathrm{R}$ and lenalidomide with or without dexamethasone had $>10 \%$ FN incidence proportions (supplemental eTable 3).

Stratified analyses of incidence proportions of FN during the chemotherapy course by age group ( $<65$ or $\geq 65$ years) and number of comorbidities $(0$, $1, \geq 2$ ) are presented in supplemental eTable 4. Clear differences were not obvious by age group or number of comorbidities examined.

Incidence proportions of grade $4(22.2 \%-52.1 \%)$ and $3 / 4$ neutropenia $(27.2 \%-62.8 \%)$ were high for all regimens investigated (Table 3 ).

\section{Discussion}

In this study, we characterized the incidence proportions of $\mathrm{FN}$ in patients who received selected chemotherapy regimens in real-world clinical practice. Using current NCCN criteria, our findings suggest that the $\mathrm{TC}$ and $\mathrm{TCH}$ regimens for breast cancer should be classified as regimens with high risk for $\mathrm{FN}$ $(>20 \%)$. There is also evidence suggesting that incidence proportions of FN for XELOX and FOLFOX6 for CRC, B $\pm \mathrm{R}$ for NHL, and lenalidomide with or without dexamethasone for $\mathrm{MM}$ were $>10 \%$.

Treatment with TC and TCH without ppG-CSF among patients with breast cancer was associated with a $20.9 \%$ and $25.1 \%$ risk of developing FN, respectively. However, the actual FN risk during the chemotherapy course was likely underestimated owing to informative censoring and misclassification of FN. Calculation of the overall incidence proportion assumes that patients who discontinued the regimen early would not have developed FN if they had continued the regimen. The higher incidence proportions of FN ( $>30 \%$ to $>40 \%$ ) observed for those who completed fewer cycles of TC or TCH suggested that upper bounds of the actual $\mathrm{FN}$ risks for TC and TCH might be much higher than $20 \%$ if the censored patients had continued the regimen. Our findings are in contrast with the low FN incidences reported in pivotal 
clinical trials for the TC and TCH regimens (5\% and $9.6 \%$, respectively), in which hematopoietic growth factors or prophylactic antibiotics were allowed. ${ }^{23,24}$ Noninterventional studies reported that incidences of $\mathrm{FN}$ associated with the $\mathrm{TC}$ and $\mathrm{TCH}$ regimens were considerably higher $(26 \%-41 \%)$ among those without ppG-CSF than those with ppG-CSF (0\%-7\%). ${ }^{11,25-27}$ Our sensitivity analysis supports the published FN risk reported in real-world clinical practice and adds further evidence that ppG-CSF significantly reduces the risk of developing $\mathrm{FN}$ for patients treated with the TC or $\mathrm{TCH}$ regimens $(<10 \%)$.

When intravenous antibiotic treatment and early patient censorship were not considered, the low FN incidence proportions $(<10 \%)$ observed in this analysis for patients with CRC treated with XELOX or FOLFOX6, NHL treated with $\mathrm{B} \pm \mathrm{R}$, and MM treated with lenalidomide with or without dexamethasone seem to concur with previous findings from clinical trials and observational studies. ${ }^{25-39}$ However, the actual incidence proportions of FN for these regimens were observed to be $>10 \%$ if the patterns of censoring inherent in the data are considered or if adjustments for misclassification of FN are made. These results help inform the use of prophylactic G-CSF for these regimens in clinical practice. However, of note, for XELOX, capecitabine was used from day 1 to 14 of each cycle. It is not recommended that G-CSF be administered concurrently with daily chemotherapy such as oral capecitabine because, although G-CSF stimulates the growth of myeloid stem cells, the chemotherapy would simultaneously kill fast-growing cells. ${ }^{40-42}$

High proportions of patients developed neutropenia even when they received regimens with nominally low FN risk (eg, XELOX, FOLFOX6, B \pm R, and lenalidomide \pm dexamethasone). Although we suspect that the true $\mathrm{FN}$ risk for these regimens is likely to be greater when accounting for censoring, it is also likely that some chemotherapy drugs suppress the bone marrow's production of neutrophils without interfering with phagocytic function or overall barrier function (skin or mucosal integrity). In such cases, there may be a high risk of neutropenia without a correspondingly high risk of infection.

Previous studies demonstrated that vulnerable patient populations such as the very elderly (aged $>65$ years), patients with multiple comorbidities, and hospitalized patients tend to have higher FN- related mortality. ${ }^{43}$ Additional research is warranted to better understand the FN-related mortality associated with specific chemotherapy regimens, especially among vulnerable populations.

Several limitations should be considered. This study was subject to selection bias (including differential censoring); significant proportions of patients receiving the $\mathrm{TC}$ or $\mathrm{TCH}$ regimens were excluded because of the use of ppG-CSF. Although no obvious differences in basic demographic and clinical characteristics were observed between patients who received and did not receive ppG-CSF in the current study population, FN risk could be confounded by unmeasured characteristics (eg, performance status), which might cause underestimation of FN risks because of the possibility that patients with a poor performance status were more likely to receive ppGCSF. Bias due to differential loss to follow-up is possible because patients who developed severe adverse events (FN or others) may have switched regimens or terminated treatment prematurely. Calculating incidence proportions of FN by number of completed cycles suggested that determining the overall FN incidence proportions in the chemotherapy course, without accounting for censoring, likely underestimated FN risks. It is noteworthy that the lack of controlling for censoring is a common limitation of previous observational studies and clinical trials that evaluated FN risks for chemotherapy regimens.

This study was also limited by the use of an electronic algorithm to capture FN (because there are no specific ICD-9 diagnosis codes for FN), which may have led to misclassifications. ${ }^{43}$ Our sensitivity analysis, in which intravenous antibiotic treatment was included as part of the FN definition, aims to account for possible FN misclassification. Also, it is possible that an administrative database is not capable of accurately identifying prophylactic antibiotic use, which might lead to further underestimation of FN risks because of unrecognized prophylaxis. This might be of concern because the rate of prophylactic antibiotic use in general practice may be higher than expected. ${ }^{44}$

Finally, the findings from this highly integrated managed care organization may not be generalizable to patients treated in other settings because of differences in practice patterns (ie, G-CSF use and who received the regimen) and patient characteristics. Calculating standardized incidence proportions using all patients with cancer treated with chemotherapy at 
FN Risk for Select Chemotherapy Regimens

KPSC helped account for differences in patient demographic characteristics; KPSC cancer cases are similar to those in the California SEER registry. ${ }^{45}$

This study has several important strengths. Data were used from a large community-based population of patients with cancer; therefore, the findings may better reflect FN risks in the real-world setting. The comprehensive clinical databases at KPSC allowed for detailed characterizations of the study population for clinical history, chemotherapy regimens, and patient outcomes.

\section{Conclusions}

Our study results suggest that the breast cancer protocols TC and TCH should be classified as regimens with a high risk for FN (>20\%), and there is strong evidence (from sensitivity analyses) suggesting that $\mathrm{B} \pm \mathrm{R}$ for NHL, XELOX for CRC, and lenalidomide with or without dexamethasone for MM have a $>10 \% \mathrm{FN}$ risk. These results help fill gaps in the literature regarding $\mathrm{FN}$ risk in real-world patient populations, thus informing the use of prophylactic $\mathrm{G}-\mathrm{CSF}$ for these regimens in clinical practice.

\section{Acknowledgments}

The authors would like to acknowledge Roberto Rodriguez, MD, for participation in this study, and Meghan Johnson, PhD, and James Balwit, MS, CMPP (both from Complete Healthcare Communications, LLC, an ICON plc company, West Chester, PA), whose work was funded by Amgen Inc., for assistance in writing this manuscript.

\section{References}

1. Lyman GH, Dale DC, Crawford J. Incidence and predictors of low doseintensity in adjuvant breast cancer chemotherapy: a nationwide study of community practices. J Clin Oncol 2003;21:4524-4531.

2. Lyman GH, Kuderer NM. Epidemiology of febrile neutropenia. Support Cancer Ther 2003;1:23-35.

3. Bhana N. Granulocyte colony-stimulating factors in the management of chemotherapy-induced neutropenia: evidence based review. Curr Opin Oncol 2007;19:328-335.

4. Lyman GH, Shayne M. Granulocyte colony-stimulating factors: finding the right indication. Curr Opin Oncol 2007;19:299-307.

5. Weycker D, Hackett J, Edelsberg JS, et al. Are shorter courses of filgrastim prophylaxis associated with increased risk of hospitalization? Ann Pharmacother 2006;40:402-407.

6. Lyman $\mathrm{GH}$, Lyman $\mathrm{CH}$, Agboola O. Risk models for predicting chemotherapy-induced neutropenia. Oncologist 2005;10:427-437.

7. Crawford J, Becker PS, Armitage JO, et al. NCCN Clinical Practice Guidelines in Oncology for Myeloid Growth Factors, version 1.2016. Accessed June 6, 2016. To view the most recent version of the guidelines, visit NCCN.org.

8. Soni A, Brufsky A, Jankowitz RC, et al. Incidence of febrile neutropenia with docetaxel plus cyclophosphamide in a university-based breast oncology clinic [abstract]. J Clin Oncol 2011;29(Suppl):Abstract 9061.

9. Truong J, Lee EK, Trudeau ME, Chan KK. Interpreting febrile neutropenia rates from randomized, controlled trials for consideration of primary prophylaxis in the real world: a systematic review and meta-analysis. Ann Oncol 2016;27:608-618.

10. Lakhanpal R, Stuart-Harris R, Chan A, et al. Docetaxel and cyclophosphamide as adjuvant chemotherapy for early breast cancer: primary prophylaxis with G-CSF is required. Breast Cancer Manag 2013;2:367-374.

11. Younis T, Rayson D, Thompson K. Primary G-CSF prophylaxis for adjuvant TC or FEC-D chemotherapy outside of clinical trial settings: a systematic review and meta-analysis. Support Care Cancer 2012;20:2523-2530.

12. Weycker D, Li X, Edelsberg J, et al. Risk of febrile neutropenia in patients receiving emerging chemotherapy regimens. Support Care Cancer 2014;22:3275-3285.

13. Crawford J, Becker PS, Armitage JO, et al. NCCN Clinical Practice Guidelines In Oncology: Myeloid Growth Factors, version 1.2017. Accessed August 21, 2017. To view the most recent version of the guidelines, visit NCCN.org.

14. Kosaka Y, Rai Y, Masuda N, et al. Phase III placebo-controlled, double-blind randomized trial of pegfilgrastim to reduce the risk of febrile neutropenia in breast cancer patients receiving docetaxel/cyclophosphamide chemotherapy. Support Care Cancer 2015;23:1137-1143.
15. Gilbar P, McPherson I, Sorour N, Sanmugarajah J. High incidence of febrile neutropenia following adjuvant breast chemotherapy with docetaxel, carboplatin and trastuzumab. Breast Cancer Manag 2014;3:327-333.

16. Goldberg RM, Sargent DJ, Morton RF, et al. Randomized controlled trial of reduced-dose bolus fluorouracil plus leucovorin and irinotecan or infused fluorouracil plus leucovorin and oxaliplatin in patients with previously untreated metastatic colorectal cancer: a North American Intergroup Trial. J Clin Oncol 2006;24:3347-3353.

17. Tournigand $\mathrm{C}$, Andre $\mathrm{T}$, Achille E, et al. FOLFIRI followed by FOLFOX6 or the reverse sequence in advanced colorectal cancer: a randomized GERCOR study. J Clin Oncol 2004;22:229-237.

18. Koebnick C, Langer-Gould AM, Gould MK, et al. Sociodemographic characteristics of members of a large, integrated health care system: comparison with US Census Bureau data. Perm J 2012;16:37-41.

19. Li Y, Klippel Z, Shih X, et al. Relationship between severity and duration of chemotherapy-induced neutropenia and risk of infection among patients with nonmyeloid malignancies. Support Care Cancer 2016;24:4377-4383.

20. Li Y, Klippel Z, Shih X, et al. Trajectory of absolute neutrophil counts in patients treated with pegfilgrastim on the day of chemotherapy versus the day after chemotherapy. Cancer Chemother Pharmacol 2016;77:703-712.

21. Chao C, Page JH, Yang SJ, et al. History of chronic comorbidity and risk of chemotherapy-induced febrile neutropenia in cancer patients not receiving G-CSF prophylaxis. Ann Oncol 2014;25:1821-1829.

22. Chia VM, Page JH, Rodriguez R, et al. Chronic comorbid conditions associated with risk of febrile neutropenia in breast cancer patients treated with chemotherapy. Breast Cancer Res Treat 2013;138:621-631.

23. Slamon D, Eiermann W, Robert N, et al. Adjuvant trastuzumab in HER2positive breast cancer. N Engl J Med 2011;365:1273-1283.

24. Jones S, Holmes FA, O'Shaughnessy J, et al. Docetaxel with cyclophosphamide is associated with an overall survival benefit compared with doxorubicin and cyclophosphamide: 7-year follow-up of US Oncology Research Trial 9735. J Clin Oncol 2009;27:1177-1183.

25. Younus J, Vandenberg T, Jawaid M, Jawaid MA. Febrile neutropenia rates with adjuvant docetaxel and cyclophosphamide chemotherapy in early breast cancer: discrepancy between published reports and community practice-an updated analysis. Curr Oncol 2012;19:332-334.

26. Rocque G, Onitilo A, Engel J, et al. Adjuvant therapy for HER2+ breast cancer: practice, perception, and toxicity. Breast Cancer Res Treat 2012;131:713-721.

27. Gilbar P, McPherson I, Sorour N, Sanmugarajah J. High incidence of febrile neutropenia following adjuvant breast chemotherapy with docetaxel, carboplatin and trastuzumab. Breast Cancer Manage 2014;3:327-333.

28. Ducreux M, Bennouna J, Hebbar M, et al. Capecitabine plus oxaliplatin (XELOX) versus 5-fluorouracil/leucovorin plus oxaliplatin (FOLFOX-6) as first-line treatment for metastatic colorectal cancer. Int J Cancer 2011;128:682-690. 
Li et al

29. Uncu D, Aksoy S, Cetin B, et al. Results of adjuvant FOLFOX regimens in stage III colorectal cancer patients: retrospective analysis of 667 patients. Oncology 2013;84:240-245.

30. Luminari S, Goldaniga M, Cesaretti M, et al. A phase II study of bendamustine in combination with rituximab as initial treatment for patients with indolent non-follicular non-Hodgkin lymphoma. Leuk Lymphoma 2016;57:880-887.

31. Vacirca JL, Acs PI, Tabbara IA, et al. Bendamustine combined with rituximab for patients with relapsed or refractory diffuse large B cell lymphoma. Ann Hematol 2014;93:403-409.

32. Friedberg JW, Cohen P, Chen L, et al. Bendamustine in patients with rituximab-refractory indolent and transformed non-Hodgkin's lymphoma: results from a phase II multicenter, single-agent study. J Clin Oncol 2008;26:204-210.

33. Robinson KS, Williams ME, van der Jagt RH, et al. Phase II multicenter study of bendamustine plus rituximab in patients with relapsed indolent B-cell and mantle cell non-Hodgkin's lymphoma. J Clin Oncol 2008;26:4473-4479.

34. Kahl BS, Bartlett NL, Leonard JP, et al. Bendamustine is effective therapy in patients with rituximab-refractory, indolent B-cell non-Hodgkin lymphoma: results from a multicenter study. Cancer 2010;116:106-114.

35. Alegre A, Oriol-Rocafiguera A, Garcia-Larana J, et al. Efficacy, safety and quality-of-life associated with lenalidomide plus dexamethasone for the treatment of relapsed or refractory multiple myeloma: the Spanish experience. Leuk Lymphoma 2012;53:1714-1721.

36. Richardson P, Jagannath S, Hussein M, et al. Safety and efficacy of singleagent lenalidomide in patients with relapsed and refractory multiple myeloma. Blood 2009;114:772-778.
37. Chanan-Khan AA, Lonial S, Weber D, et al. Lenalidomide in combination with dexamethasone improves survival and time-to-progression in patients $\geq 65$ years old with relapsed or refractory multiple myeloma. Int J Hematol 2012:96:254-262.

38. Hou J, Du X, Jin J, et al. A multicenter, open-label, phase 2 study of lenalidomide plus low-dose dexamethasone in Chinese patients with relapsed/refractory multiple myeloma: the MM-021 trial. J Hematol Oncol 2013;6:41.

39. Zonder JA, Crowley J, Hussein MA, et al. Lenalidomide and highdose dexamethasone compared with dexamethasone as initial therapy for multiple myeloma: a randomized Southwest Oncology Group trial (S0232). Blood 2010;116:5838-5841.

40. Neulasta [package insert]. Thousand Oaks, CA: Amgen Inc.; 2016.

41. Chabner B, Longo DL. Cancer Chemotherapy and Biotherapy: Principles and Practice, 5th ed. Philadelphia, PA: Wolters Kluwer Health/Lippincott Williams \& Wilkins; 2011.

42. Meropol NJ, Miller LL, Korn EL, et al. Severe myelosuppression resulting from concurrent administration of granulocyte colony-stimulating factor and cytotoxic chemotherapy. J Natl Cancer Inst 1992;84:1201-1203.

43. Kuderer NM, Wolff AC. Enhancing therapeutic decision making when options abound: toxicities matter. J Clin Oncol 2014;32:1990-1993.

44. Culakova E, Poniewierski MS, Wolff DA, et al. The impact of chemotherapy dose intensity and supportive care on the risk of febrile neutropenia in patients with early stage breast cancer: a prospective cohort study. Springerplus 2015;4:396.

45. Cancer Registry of Greater California. Available at: http://crgc-cancer.org. Accessed September 13, 2016

\section{See JNCCN.org for supplemental online content.}

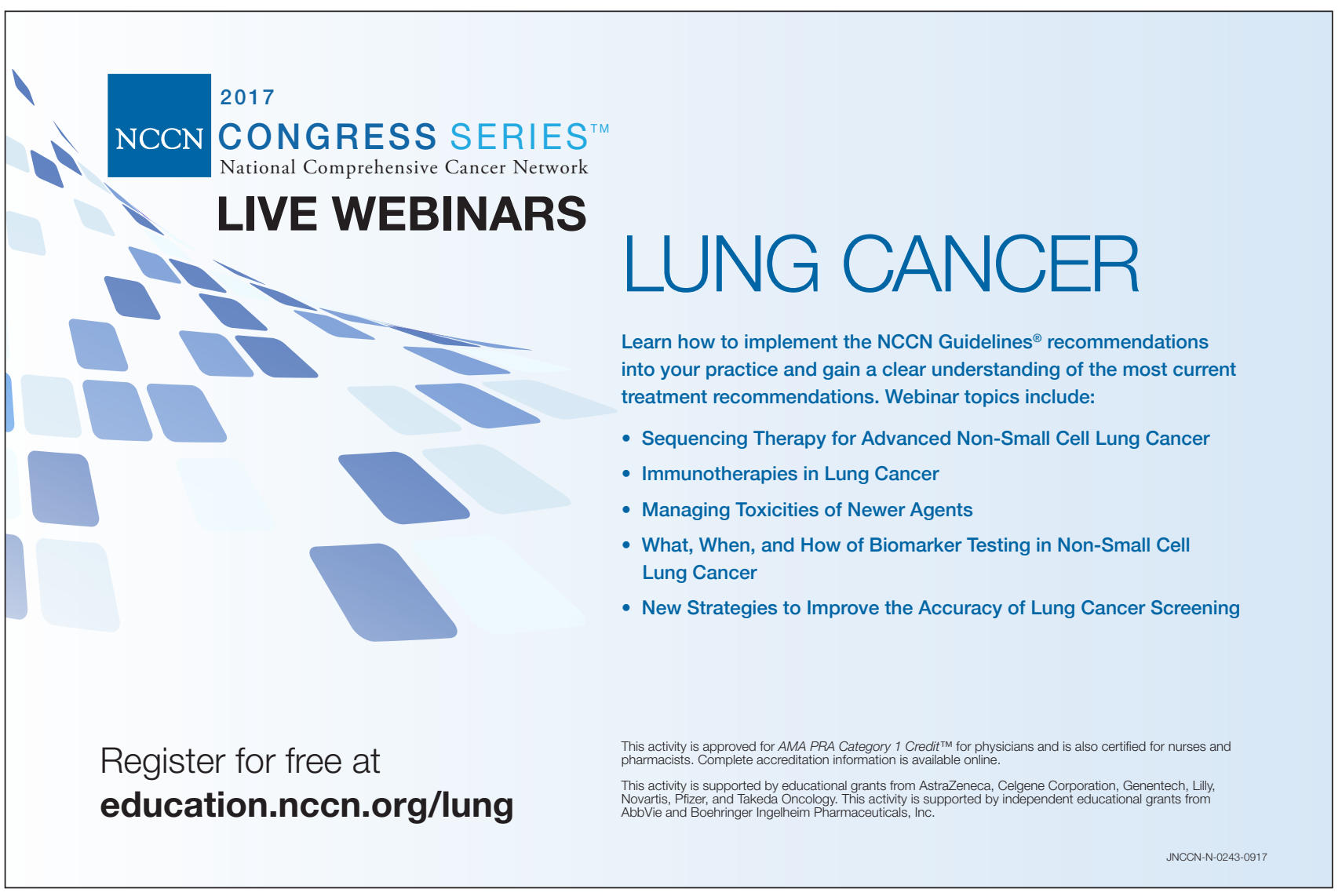

